\title{
La alfabetización desde una perspectiva crítica: Los aportes de Vygotski, Freire y Martín Baró
}

Literacy from a critical perspective:

The contributions of Vygotsky, Freire and Martín Baró

\author{
Volumen 19, Número 1 \\ Sección Especial \\ Enero-Abril
}

pp. 1-25

Este número se publica el 1 de enero de 2019

DOI: 10.15517/aie.v19i1.35569

Wanda C. Rodríguez Arocho

Revista indizada en REDALYC, $\underline{\text { SCIELO }}$

Revista distribuida en las bases de datos:

LATINDEX, DOAJ, REDIB, IRESIE, CLASE, DIALNET, SHERPA/ROMEO, QUALIS-CAPES, MIAR

Revista registrada en los directorios:

ULRICH'S, REDIE, RINACE, OEI, MAESTROTECA, PREAL, CLACSO 


\title{
La alfabetización desde una perspectiva crítica: Los aportes de Vygotski, Freire y Martín Baró
}

\author{
Literacy from a critical perspective:
}

The contributions of Vygotsky, Freire and Martín Baró

\section{Wanda C. Rodríguez Arocho'}

\begin{abstract}
Resumen: Este texto tiene su origen en una conferencia dictada en el X Encuentro Vygotski, Freire y Martín Baró: Memoria y Vigencia en Movimiento, organizado por el Instituto de Investigación Educativa de la Universidad de Costa Rica. El mismo es una reflexión teórica que tiene como propósito sostener que, pese a que las producciones intelectuales y las acciones sociopolíticas de Lev S. Vygotski, Paulo Freire y Ignacio Martín Baró se originaron en momentos y contextos históricos distintos, sus ideas sobre las relaciones entre lenguaje, conciencia, pensamiento, afecto y actividad humana tienen vigencia y pertinencia para aproximarnos a la alfabetización crítica en la actualidad. Luego de una breve introducción al tema, prosigo con un análisis de la alfabetización como actividad históricamente situada, culturalmente mediada y socialmente ejecutada, que pone de manifiesto la intrincada relación entre tecnologías, alfabetización y transformación psicosocial. A partir de ese análisis, paso a precisar lo que cada uno de los autores mencionados aportó a la comprensión de dicha relación. Continúo con un examen de la vigencia y el movimiento de su legado y sus implicaciones para la práctica educativa orientada a la alfabetización crítica. Finalizo con una conclusión general.
\end{abstract}

Palabras clave: alfabetización crítica, Lev S. Vygotski, Paulo Freire, Ignacio Martín Baró

Abstract: This text originated as a lecture given at the $X$ Encuentro Vygotski, Freire y Martín Baró: Memoria y Vigencia en Movimiento, organized by the Institute of Educational Research at the University of Costa Rica. It is a theoretical reflection to argue that, although the intellectual productions and the sociopolitical actions of Lev S. Vygotsky, Paulo Freire and Ignacio Martín Baró originated in different historical moments and contexts, their ideas about the relations between Language, conscience, thought, affection and human activity are valid and pertinent to approach critical literacy at present. After a brief introduction to the subject, I proceed with an analysis of literacy as a historically situated, culturally mediated and socially executed activity that highlights the intricate relationship between technologies, literacy and psychosocial transformation. From that analysis, I go on to specify what each of the authors alluded authors contributed to the understanding of this relationship. I continue with an examination of the validity and movement of their particular legacy and the implications for an educational practice oriented to critical literacy. This leads to a final general conclusion.

Key Words: critical literacy, Lev S. Vygotsky, Paulo Freire, Ignacio Martín Baró

\footnotetext{
${ }_{1}$ Profesora Jubilada de la Universidad de Puerto Rico, Recinto

Río Piedras, Puerto Rico.
}

Dirección electrónica: wandacr@gmail.com

Documento recibido: 9 de julio, 2018

Enviado a corrección: 12 de octubre, 2018

Aprobado: 29 de octubre, 2018 


\section{Introducción}

Este trabajo es una reflexión sobre los aportes particulares de Lev S. Vygotski, Paulo Freire e Ignacio Martín Baró a una perspectiva crítica de la alfabetización. A pesar de que sus vidas y obras se dan en diferentes contextos y momentos, sus producciones intelectuales ofrecen ideas para elaborar una red conceptual que permite entender y explicar los actos de leer y escribir como prácticas con capacidad transformadora sobre los procesos mentales propiamente humanos. Dicha red puede orientar los métodos didácticos para la alfabetización crítica, así como el estudio de las actividades de enseña-aprendizaje que la promueven.

La preocupación actual por el rol de los medios de comunicación masiva en la elaboración y difusión de las llamadas noticias falsas, las narrativas de realidades alternativas y su impacto en las condiciones sociopolíticas que constituyen parte del entorno de vida ponen de manifiesto la importancia y pertinencia del problema que nos ocupa. Vivimos en un mundo en que la cultura del papel coexiste con la cibercultura, con modos diferentes de leer, de escribir y de pensar en cada una. Pese a la diferencia en modalidades, la alfabetización puede practicarse acrítica o críticamente en ambas. Esta última se ancla se orienta al pensamiento crítico. Rojas Osorio (2000) ha identificado cinco dimensiones: lógica, sustantiva, contextual, dialógica y la pragmática. A estas dimensiones Vygotski, Freire y Martín Baró agregan la éticas y la política. Antes de examinar sus aportes particulares a la elaboración de una perspectiva crítica de la alfabetización, procede examinar el sentido histórico de esa práctica sociocultural.

\section{Tecnologías, transformación social y alfabetización crítica}

En el texto Del hacha al chip: Cómo la tecnología cambia nuestras mentes, Burke y Ornstein (1995) utilizaron hallazgos de la antropología cultural para argumentar que la fabricación de herramientas en el curso de la evolución de nuestra especie propició tanto la transformación del entorno de vida como la fabricación de esperanzas y sueños. Estos autores argumentan que ese proceso de invención, creación y utilización de herramientas ha resultado en cambiantes configuraciones del mundo que habitamos, "las creencias por las que luchamos y morimos, los valores de los que nos nutrimos, y nuestra propia naturaleza" (p. 15). Esto convierte la consideración de la creación y el uso de tecnologías en indispensables para comprender y explicar la mente humana en su complejidad. La dimensión afectiva implicada en creencias, valores y subjetividad trasciende el énfasis 
predominantemente cognitivo que ha dominado los abordajes del estudio del impacto de las tecnologías en las transformaciones históricoculturales de la mente humana. En este contexto, el legado Vygotski, Freire y Martí Baró, al análisis de los temas antes señalados, tiene vigencia y guía movimientos individuales y sociales por la integración que hicieron entre pensamiento y afectividad.

El momento actual se caracteriza por una tendencia en diversos campos del saber a buscar una comprensión compleja de la relación entre cognición y afectividad. Investigaciones recientes documenta las transformaciones subjetivas e intersubjetivas vinculadas a las nuevas tecnologías de la información y la comunicación (TIC) (Clark,2003; Figueroa Sarriera, 2017; Sabila, 2009). Esa discusión, con frecuencia, deja de lado que las viejas tecnologías, utilizadas históricamente para compartir información y comunicarnos, también resultaron en configuraciones mentales que en estos momentos coexisten con las configuraciones actuales (Flores, 2018). Una mirada histórica, social y cultural a la relación entre tecnologías y mentalidades puede resultar muy útil al enfrentar los retos que presenta la alfabetización crítica a la psicología y la educación contemporáneas.

La alfabetización ofrece una instancia privilegiada para examinar el poder, la fuerza y el alcance de una herramienta cultural como mediadora en la conformación de la subjetividad, sea en su variante tradicional o en su variante digital. Aunque aprendemos a leer y a escribir sin reflexionar sobre su origen y transformaciones, la alfabetización es una práctica social con variadas expresiones históricas. En su variante tradicional, la alfabetización, junto a la agricultura y al urbanismo, se ha mencionado como uno de los principales propulsores de cambio en la historia cultural de la humanidad y en la configuración psicológica (Olson y Cole, 2006). Primero fue la tecnología la que permitió comunicar experiencias e ideas mediante dibujos y símbolos. La utilización de instrumentos, como cinceles, tinta y lápices para hacer marcas en papel y otras superficies, fue determinante en la emergencia y configuración de procesos cognitivos complejos y nuevos usos del lenguaje (Olson, 1994; Flores, 2018).

En el mundo occidental, desde sus inicios, la tecnología que dio paso a la actividad alfabetizadora ha estado vinculada al ejercicio del poder político y religioso. Al comienzo, el acceso a la escritura se limitaba a religiosos y a nobles, y los textos que producían se circunscribían a temas religiosos, históricos y legales (Olson, 1994; Flores, 2018). Esta situación cambió con el advenimiento de otra innovación tecnológica: la imprenta. Con la imprenta se rebasaron los límites del acceso a los textos y se hicieron posibles procesos de 
transformación sociocultural, como por ejemplo, la Reforma. Al mismo tiempo, se abrió camino para la creación y socialización de nuevas formas de escritura evidentes en la proliferación de géneros literarios. La alfabetización masiva fue instrumental en la creación de un nuevo orden social, pero su enseñanza y aprendizaje se realizó sin considerar que tanto la palabra escrita como la hablada van cargadas de expresiones culturales que encubren ideologías.

La alfabetización fue entendida, principalmente, como una actividad orientada a la comprensión de caracteres gráficos, un acto de descifrar el código y entender su significado. La alfabetización tradicional se centró en la adquisición de destrezas para codificar y decodificar (con énfasis en la fonética y en la ortografía), y para procesar información secuencialmente y dominar la gramática. Como práctica social, la alfabetización tradicional se asume como neutral, por lo que se ejecuta de forma similar independientemente de la lengua, la cultura y la ubicación del hablante en la organización social en que vive. Esto permite explicar por qué aprendemos a leer y escribir sin conocer la historia de la alfabetización y las transformaciones socioculturales atadas a ella (Flores, 2018). También, contribuye a entender por qué en esos aprendizajes la clase social, el género, la raza, la etnia, el credo y otras ideas del autor o la autora son consideradas (en el mejor de los casos) una guía para comprender y significar la narración. Es decir, las coordenadas para comprender y significar están dadas por una voz de autoridad, el alfabetizador o la alfabetizadora. Como consecuencia, se obstaculiza la toma de conciencia de las dimensiones históricas, culturales y sociales de la lecto-escritura.

La situación descrita generó agudas críticas en la educación y en la sociolingüística desde la década de 1970, y abrió camino a la pedagogía crítica (Bernstein, 1971; Freire, 1971, 1972, 1991; Freire y Macedo, 1987; Kincheloe, 2008). Pese a ello, la descontextualización y automatización de los actos y escribir se extrapolaron a la alfabetización digital. Como en la alfabetización tradicional, en la alfabetización digital aprendemos destrezas con poca conciencia del origen de la tecnología que empleamos y de su impacto con respecto a los que hacemos y somos. Esto, pese a que la investigación contemporánea ha documentado transformaciones psicológicas significativas en las personas que utilizan rutinariamente las nuevas TIC (Rodríguez Arocho, 2018). Al mismo tiempo, el impacto de estas tecnologías en la economía, la política y otras instancias de interacción social ha sido igualmente documentado por las ciencias sociales contemporáneas (Colombo y Fortunati, 2011). 
En el contexto de lo expuesto, es pertinente plantear la falta de alfabetización crítica como un problema fundamental en la educación contemporánea, tanto la que se orienta por la cultura del papel como la que se orienta por la cibercultura. Este problema está marcado por dos consideraciones relacionadas: 1) la carencia de reflexividad histórico-cultural con respecto a la relación entre las herramientas que utilizamos para realizar una determinada actividad (como leer, escribir, hablar) y la actividad misma, y 2) la escasa comprensión de que nuestra psique se configura en las complejas dinámicas de esa relación. Las obras de Vygotski, Freire y Martín Baró ofrecen pautas orientadoras para abordar estos problemas.

\section{Aportes clave}

\subsection{El aporte de Lev S. Vygotski}

Lev S. Vygotski nació en Bielorrusia en 1896 y murió en Moscú en 1934. Su vida y su obra están ligadas ineludiblemente a los antecedentes y consecuencias de la Revolución Rusa de 1917. Varios autores han examinado la relación entre la biografía de Vygotski y el contexto históricosocial de su producción (Blanck, 1990; Van der Veer y Valsiner, 1991; Yaroshevsky, 1989). Vygotski inició su vida profesional como docente, y los temas relacionados con la enseñanza y el aprendizaje le interesaron hasta el final de su vida.

Además de ocuparse de elaborar una compleja propuesta teórica para explicar el desarrollo de las funciones mentales asociadas con la conciencia (Vygotski, 1993, 1995), se ocupó de investigar varios problemas con implicaciones prácticas, como el desarrollo de funciones mentales complejas mediadas por la educación, la evaluación psicológica, la educación para personas con necesidades especiales para propulsar su desarrollo y la alfabetización (Kozulin, Gindis, Ageyev y Miller, 2003). Su obra ha generado una considerable producción de interpretaciones, análisis, debates y un cuerpo de elaboraciones conceptuales (Esteban-Guitart, 2016; Esteban-Guitart y Moll, 2014a, 2014b; González Rey, 2011; Moll, 2014; Yatsnisky, Van der Veer y Ferrari, 2014).

Para entender la importancia de la alfabetización para Vygotski es necesario comprender el rol que le dio en el desarrollo de funciones mentales superiores. El aporte principal de Vygotski (1995) fue elaborar una teoría del desarrollo psicológico que lo concibe como el producto emergente de condiciones históricas, sociales y culturales, al tiempo que lo funde con la acción educativa (Daniels, 2001; Kozulin, Gindis, Ageyev y Miller, 2003; Moll, 1990). De acuerdo con Bruner (1962, 1987), esta teoría del desarrollo psíquico es, al mismo tiempo, una teoría de la educación. La tesis central de la teoría es que la mente se conforma 
y transforma en el curso de prácticas e interacciones sociales que son mediadas por herramientas creadas en la historia cultural para enfrentar las condiciones del ambiente. La educación juega un rol fundamental en ese proceso al punto de ser considerada la quintaesencia de la mediación cultural (Moll, 1990).

Un aporte importante en la elaboración de esta tesis es la idea de dos tipos de herramientas: los instrumentos materiales, que permiten las acciones para transformar el medio externo; y los instrumentos psicológicos (signos y símbolos), que permiten la ejecución de procesos internos. Entre estos últimos, otorgó un rol privilegiado al lenguaje, tanto hablado como escrito. Los niveles de conocimiento y apropiación de ambos tipos de instrumentos se desarrollan mediante procesos de aprendizaje en contextos específicos de actividad. Uno de esos contextos es la institución escolar, donde ocurren las acciones alfabetizadoras. Para Vygotski esas acciones implican un proceso complejo de significación y elaboración de sentido.

En su obra Pensamiento y lenguaje (1993), Vygotski dedica particular atención al proceso de aprendizaje de la lectura y la escritura y al examen de las consecuencias de ese proceso en la formación y configuración de funciones mentales complejas como la atención voluntaria, la memoria lógica, el razonamiento, la solución de problemas y el ejercicio de la voluntad. Consideró que la expresión de estas funciones está vinculada a la formación de conceptos, los cuales caracterizó como unidades de pensamiento que adscriben significado al mundo al proveer generalizaciones que sirven para darle orden. Consideró que la formación de estos conceptos no sigue un orden lineal ni definido, sino uno zigzagueante en función del contacto con otros conceptos que se adquieren en la medida en que la experiencia y el conocimiento se amplifican.

Vygotski planteó una compleja dinámica entre los conceptos espontáneos que se adquieren en la vida diaria y los conceptos científicos que se aprenden en la escuela. Propuso que el primero ofrece las herramientas para el manejo de la realidad al nivel de lo concreto y específico en nuestra cotidianidad. Al mismo tiempo, argumentó que la escuela introduce conceptos y categorías producidas por los saberes disciplinados. Vygotski se refirió a estos como conceptos científicos, ya que siguen las reglas de la lógica que guían la actividad científica y reflejan el desarrollo cultural. Los conceptos espontáneos y los científicos interactúan en complejos procesos de significación que son particularmente importantes en la lectura y la escritura. Gracias a esos procesos, el texto impreso abre 
posibilidades de reflexión sobre el contenido y la forma de lo dicho, búsquedas de relaciones intertextuales, indagación dialógica y atribución de sentido (Smagorinsky, 2011).

Los desarrollos actuales de la perspectiva vygotskiana enfocan tres aspectos que es pertinente destacar. El primero se refiere a la necesidad de trascender una lectura simplista de Vygotski que, por un lado, ha llevado a que equivocadamente se le clasifique como cognitivista (Rodríguez Arocho, 2013) y, por otro lado, ha fomentado distorsiones de conceptos claves, como es el caso de la zona de desarrollo próximo (Rodríguez Arocho, 2015). El énfasis en el funcionamiento cognitivo ha obviado o minimizado el rol de la afectividad en la actividad en la acción humana. En el último capítulo de Pensamiento y Lenguaje, Vygotski (1934) hace claro que el lenguaje y el pensamiento están atravesados por motivos, deseos e intenciones, y que solo considerando la dimensión afectiva puede comprenderse el pensamiento construido y expresado en palabras. Esta complejidad se relaciona con el segundo aspecto mencionado. La zona de desarrollo próximo trasciende la relación personal entre quien enseña y quien aprende bajo su guía o con la ayuda de un par más capaz. Esas interacciones están atravesadas por particulares condiciones de vida (explotación, pobreza, marginalización o sus opuestos e intermedios) y las particulares situaciones de desarrollo vinculadas (Rodríguez Arocho, 2015).

El tercer aspecto en los desarrollos actuales también implica un movimiento a la complejidad. Este tiene ver con que la división entre herramientas materiales y semióticas, es más compleja de lo que Vygotski pensó. Lo anterior es particularmente pertinente cuando se consideran las transformaciones psíquicas asociadas con nuevas tecnologías de la información y la comunicación. En intento de atender esta situación, Cole (1996) plantea que la noción de artefacto tiene mayor utilidad conceptual porque permite analizar el proceso de adquisición y uso de la herramienta material, su transformación en instrumento psicológico individual y su lugar en las narrativas e imaginarios de época. Esta elaboración parece ser más útil en el análisis de las transformaciones mentales asociadas a las nuevas tecnologías de la comunicación y la información (TIC) y de la alfabetización digital. La elaboración no contradice, sino que apoya la idea de que los procesos mentales se conforman mediante el uso de creaciones humanas de orden material y simbólico. Por lo tanto, cualquier análisis de similitudes y diferencias en funciones mentales debe considerar las mediaciones culturales que le dan forma.

Buena parte de las investigaciones de Vygotski, sus colaboradores y colaboradoras estuvo orientada a examinar las transformaciones y la evolución de esas funciones en el 
curso del desarrollo desde la infancia a la adolescencia (Vygotski, 1991; Vygotski y Luria, 1994). De los hallazgos de esas investigaciones, surgió la conclusión de que la educación, tanto informal como formal, es la forma por excelencia en la apropiación o uso de esas producciones o herramientas culturales (instrumentales y semióticas), artefactos e instrumentos psicológicos en diversos acercamientos a la teoría de la sociogénesis de las funciones mentales propuesta por Vygotski (Cole, 1996; Kozulin, 2000). En esta conclusión, el alfabeto resalta como una de las producciones culturales más significativas en la historia de la humanidad y las acciones alfabetizadoras como centrales en la formación de la conciencia, la cual implica funciones psicológicas complejas.

Para Vygotski y Luria (Luria, 1976), el papel de la alfabetización (y la subsecuente escolarización) en el desarrollo de funciones psicológicas complejas, como la percepción, la atención voluntaria, la memoria lógica, procesos de generalización, abstracción, deducción, inducción, razonamiento y solución de problemas, la solución de problemas, imaginación, autoconciencia y autoanálisis fueron objeto específico de investigación. Llegaron a esta conclusión como resultado de varios estudios en torno a los efectos de la alfabetización y la escolarización en la estructura y las funciones cognitivas de campesinos iliteratos en el Asia Central. Estos estudios fueron objeto de variadas críticas en su momento histórico y sigue siendo debatido en la actualidad (Lamdan y Yasnitsky, 2016; Van der Veer y Valsiner, 1991). Las críticas se enfocan en interpretaciones alternativas y omisiones en relación con los datos disponibles, pero sobre todo en que los hallazgos implican devaluación del pensamiento no alfabetizado y declaración de superioridad de unas formas de conocimiento sobre otras.

Algunas de esas críticas argumentan un sesgo que desvaloriza las formas de pensamiento que no se ajustan a los estándares occidentales que han exaltado las capacidades lingüísticas y el razonamiento abstracto como las definitorias de la inteligencia humana. Otras críticas apuntan a una falta de profundización en el análisis de la configuración de los procesos cognitivos estudiados, particularmente, la consideración de otras variables interventoras. La respuesta contestataria a las críticas ha generado producciones que buscan aproximaciones teóricas y metodológicas con mayor elaboración que las utilizadas por Vygotski y Luria (Lee y Smagorinsky, 2000; Smagorinsky, 2011).

En la exposición que antecede se aprecia vigencia y movimiento en la aproximación vygotskiana al tema general de la mediación cultural y al tema específico de la alfabetización. Resalta la idea de una relación entre la alfabetización, las herramientas utilizadas para construir conocimiento y las formas de conciencia. Estas ideas se potencian en el legado de 
Paulo Freire (Rodríguez Arocho, 2000), cuyos rasgos más sobresalientes presentamos a continuación.

\subsection{El aporte de Paulo Freire}

Paulo Freire nació en Recife, Brasil, en 1921 y murió en Sao Paulo en 1997. Estudiosos de su biografía subrayan que Recife, la capital del estado de Pernambuco, es una de las zonas de mayor pobreza en el país más grande de América Latina. Sus biógrafos Gerhardt (1993) y Kirylo (2011) destacan el impacto que dicha pobreza y las condiciones que la propiciaban y mantenían tuvieron en el desarrollo del pensamiento de Freire. A pesar de haber tenido condiciones de crianza de clase media, desde muy temprano en su vida, Freire se interesó por la educación de las poblaciones pobres en su país. Tras haber obtenido un grado académico en derecho, realizado y laborado en sindicatos, orientó su trabajo a la educación con propuestas novedosas y con sello radical. Desarrolló un método para alfabetizar enraizado en el contexto y las vivencias de los sujetos, el cual fue aplicado con éxito en Brasil y otros países. Con el materialismo histórico interpretado desde una postura crítica, analizó los problemas de la educación tradicional y su rol en la domesticación del pensamiento. Como resultado de ese análisis, denunció públicamente la pedagogía del oprimido y creó las condiciones para una pedagogía de la emancipación y la esperanza.

En su proceso de formación formal entró en contacto con autores que influirían en su pensamiento y en su obra. En sus vivencias, entró en contacto con experiencias y discursos que le permitieron un diálogo permanente entre teoría y práctica. Aunque su formación religiosa, influenciada principalmente por su madre, fue en el catolicismo tradicional, eventualmente, asumió posiciones críticas en sincronía con la teología de la liberación (aunque en el momento no se le llamara así). Sufrió prisión en dos ocasiones en su propio país, sufrió el exilio, tuvo vivencias en otros contextos y salió de esto fortalecido. Al momento de su muerte, su obra había sido traducida a 18 idiomas. Sus ideas continúan en movimiento. De la extensa producción de Freire, como de la de Vygotski, solo destacaré las que parecen más pertinentes al tema de la alfabetización crítica.

La aproximación de Freire al problema de la alfabetización es revolucionaria en el sentido de enfrentar la idea tradicional de la lectoescritura como una técnica neutral orientada al desarrollo del pensamiento. El entramado conceptual que construyó incluye nociones como praxis, problematización, concienciación, temas generadores, educación bancaria, educación liberadora. Además, subraya una compleja dinámica entre lenguaje y 
conciencia. Como en el caso de Vygotski, los biógrafos de Freire resaltan la relación entre sus vivencias personales y el contexto sociohistórico de su producción intelectual. Enfocan de manera particular sus relaciones intertextuales con diversidad de autores en su proceso formativo y las relaciones dialógicas marcaron su quehacer.

Gerhardt (1993) destaca el aporte epistemológico de Freire, particularmente en relación con la transitividad crítica. Plantea que en este aporte se resumen varios postulados: “a) el desarrollo y ejercicio de una conciencia crítica es el producto del "trabajo educativo crítico"; b) la tarea de la educación como instrumento del proceso del desarrollo de la conciencia depende de dos actitudes y actividades básicas: la crítica y el diálogo; c) una conciencia crítica es característica de las sociedades que poseen una verdadera estructura democrática." (p. 470). Estos postulados, como los de Vygotski con respecto al efecto de la alfabetización sobre las formas complejas de pensamiento, descansan en una perspectiva que concibe al ser humano como capaz de razonar, comprender y transformar sus condiciones de vida y, con ello, transformarse a sí mismo. Ambos entendieron que esos procesos no son generados espontáneamente, sino que hay fuerzas de diferente naturaleza que los influencian y condicionan. Igualmente, entendieron que la educación puede y debe jugar un papel definitivo en la formación y desarrollo de los procesos mentales y tomar en cuenta las particulares situaciones sociales de vida de los sujetos. Estas situaciones son definidas por una dinámica entre las condiciones materiales de vida y las relaciones interpersonales en el entorno sociocultural. Esas relaciones incluyen desde los vínculos afectivos hasta formas de ejercicio del poder.

La centralidad de las consideraciones en el párrafo anterior se hace evidente en el método de alfabetización diseñado por Freire y contribuye a entender la efectividad de este. Dicho método toma como punto de partida la situación particular de los sujetos, e implica un aprendizaje totalmente situado y contextual. Este método, que continúa siendo utilizado (Ojokheta, 2016) se desarrolla en varias fases. La primera fase implica estudio y comprensión del contexto en que ocurre la acción alfabetizadora. Mediante diálogos con los integrantes del grupo o comunidad, los alfabetizadores entran en contacto con las palabras que la población, a ser alfabetizada, utiliza en su realidad cotidiana. La segunda fase implica la selección de algunas palabras entre las encontradas en los diálogos. La selección se hace a partir de un criterio claro: la palabra tiene que ser significativas para el hablante, lo cual implica que posee una carga emocional. Esta fuerza emocional de los vocablos los convierte en lo que Freire llamó palabras generadoras, por su capacidad de llevar a la producción de 
otras palabras. La tercera fase y última fase consiste en el proceso de alfabetización propiamente. Esta fase se descompone en tres movimientos: sesiones de motivación, desarrollo de materiales didácticos y aprendizaje de la lectura, el cual implica codificación y decodificación. El proceso reconoce, desde el comienzo, la unidad sujeto-contexto.

Como estrategias didácticas la palabra generadora, la codificación y la decodificación movilizan una progresiva capacidad no solo para leer la palabra, sino para atribuirle sentido personal y social. Para Freire, el acto de leer no es meramente decodificar lo escrito en un texto, sino entender la relación que la palabra guarda con el mundo en que se vive. En ese mundo, las memorias y las imaginaciones del sujeto ocupan un lugar en el que lo afectivo y lo cognitivo se funden. La alfabetización crítica parte del entendido de una intrincada relación entre sujeto, texto y contexto, que debe ser parte esencial en la enseñanza-aprendizaje de la lectoescritura. Entendida en estos términos, la alfabetización crítica propicia dos procesos esenciales en la pedagogía liberadora de Freire: problematización y concienciación.

Freire concibió la problematización como una estrategia para el cuestionamiento de lo que se considera evidente y se naturaliza, es decir, deja de pensarse como una hechura humana. La estrategia consiste en la interpelación de las palabras y en un diálogo inquisitivo de sus significados. La estrategia se ata a los contextos de vida y trabajo. Este diálogo con otros, con respecto al objeto o proceso problematizado, es fundamental. La problematización lleva a develar las fuerzas y dinámicas de la opresión y es una condición necesaria para la concienciación y la acción transformadora. Como resultante del proceso de problematización, vinculado a la alfabetización crítica, emerge la concienciación. Este proceso de toma de conciencia implica percibir y reflexionar sobre las causas y razones de las desigualdades sociales, políticas y económicas, tomar posiciones y actuar frente a ellas.

La idea de la educación problematizadora como herramienta fundamental para la concienciación y la praxis se fundamenta en el entendido de que el lenguaje que manejamos no es neutral, sino que porta cargas semánticas de naturaleza ideológica que su método alfabetizador persigue revelar. Para Freire (1972), “[...] la educación problematizadora implica un acto permanente de develación de la realidad. La educación bancaria busca la inmersión; la liberadora, la emersión. Este debe ser el resultado de la inserción crítica en la realidad“" (p. 92).

En su análisis de las contribuciones de Vygotski a la educación, Lake (2012) destaca su sincronía con la propuesta de pedagogía crítica de Freire. En particular, resalta la visión común en cuanto a los procesos de significación, el aprendizaje basado en el planteamiento 
y la solución de problemas de interés común, el diálogo y la colaboración. No es coincidencia que en su trabajo de pedagogía crítica, Kincheloe (2008) también subraye las ideas compartidas como señas de identidad de esta, y que autores contemporáneos se ocupen de su análisis crítico (Villacañas de Castro, 2016).

El acto de develación necesario para la concienciación implica una actitud de sospecha y resistencia a formas de opresión, y es piedra angular en la pedagogía crítica que Freire origina. Implica también la necesidad de reconocer las dimensiones ideológicas, políticas y éticas de las prácticas sociales, entre las que destacan las prácticas educativas. Estas dimensiones fueron objeto de interés, estudio y trabajo en la obra de Ignacio Martín Baró, quien hizo referencias directas en su obra al legado de Vygotski y de Freire.

\subsection{El aporte de Ignacio Martín Baró}

Ignacio Martín Baró nació en Valladolid, España, en 1942 y murió asesinado en San Salvador, El Salvador, en 1989 donde vivió experiencias atadas a la guerra civil en el país y sus consecuencias. En 1959 entró como novicio a la Compañía de Jesús de Orduña y fue sacerdote jesuita hasta su muerte. Luego de un bachillerato en filosofía y una licenciatura en filosofía y letras en la Universidad Javeriana en Bogotá, Colombia, su educación formal culmina con un doctorado en 1979 en psicología social y organizativa en la Universidad de Chicago en los Estados Unidos de América. Antes y después de completar su doctorado ocupó posiciones docentes y directivas en la Universidad Centroamericana José Simeón Cañas (UCA) en San Salvador.

Hacia el final de su vida, elaboró su propuesta para una psicología de la liberación (Martín Baró, 1986) en la que sobresalen varios conceptos claves como sujeto histórico, liberación, ideología, desideologización, realismo crítico y concienciación, algunos de los cuales ya había trabajado (Martín Baró, 1983). Montero (1991) se refirió a la psicología de la liberación como una teoría sociopolítica que se afianza en una psicología social crítica. Esa misma autora ha apuntado como influencias, en la obra de Martín Baró, el marxismo, la teoría crítica, la teología de la liberación y la obra freiriana (Montero, 2004).

Su intelecto y su sensibilidad le acercaron al análisis de problemas vinculados a la pobreza y la violencia de Estado, y a una identificación con las masas sufridas del Salvador. Su denuncia y su posicionamiento ético-político y su compromiso de trabajo, junto al de varios de sus compañeros jesuitas, resultó en su ejecución por el ejército salvadoreño junto a sus compañeros sacerdotes Ignacio Ellacuría, Segundo Montes, Juan Ramón Moreno 
(españoles como él) y Joaquín López (salvadoreño), además de Elba Ramos, empleada de la residencia donde vivían en el campus universitario, y su hija, Celinda de 16 años (ambas salvadoreñas). La UCA se ha ocupado de mantener vivo su legado en un portal digital de libre acceso con sus obras. La vigencia y el movimiento de su legado en América Latina se ha documentado, analizado (Dobles Oropeza, 2016; Montero, 1991, 2004), Portillo, 2012) y ha trascendido las fronteras regionales, como demuestran los trabajos de Amalio Blanco en España, Mark Burton en Inglaterra y Tod Sloan en Estados Unidos de Norteamérica, entre los de mayor difusión. En este último país, Aaron y Corne (1994) publicaron una selección de sus trabajos sobre psicología de la libración en inglés.

La orientación de Martín Baró (1986) hacia una psicología de la liberación articula, al mismo tiempo, una crítica a la profunda crítica a la psicología social de su tiempo y un diseño para su transformación en su época. En esta crítica y la propuesta contestataria encontramos ecos del análisis que hizo Vygotski (1991) en su ensayo El sentido histórico de la crisis de la psicología. Ambos coincidieron en el diagnóstico de que una psicología que no considerara lo histórico, lo social y lo cultural en su complejidad era incapaz de entender y explicar las acciones propiamente humanas.

Para Martín Baró construir una psicología de la liberación exigía primero una liberación de la psicología. Elaboró que la psicología debe liberarse de lo que llamó "el mimetismo cuentista", en referencia a la aceptación y uso irreflexivo y acrítico de teorías y modelos producidos en realidades distintas. Argumentó que esta aceptación constituye una negación del carácter sociohistórico de la producción de conocimiento y abogó por una epistemología que superara el positivismo, el individualismo, el hedonismo, la visión homeostática y el ahistoricismo. Concluyó que esto resulta en la confección de explicaciones que simplifican y distorsionan la realidad, para la elaboración de ideologías y para la ejecución de prácticas que naturalizan complejas dinámicas sociales, haciéndolas invisibles o difíciles de develar. Propuso que estas explicaciones deben elaborase desde un realismo crítico, cuyos antecedentes y planteamientos han sido objeto de análisis por Blanco, de la Corte y Sabucedo (2018).

La noción de realismo crítico es importante porque coloca la indagación sobre la historia social como punto de partida de la investigación psicológica. Es la realidad vivida la que debe guiar la pauta en la formulación del problema y en la comprensión. Esta idea es análoga a la que utilizó Freire en su método de alfabetización, al proponer las propias palabras de los sujetos como punto de inicio en el proceso de entender sus significados y la 
relación de estos con las condiciones de existencia. También armoniza con la idea que plantearon Vygotsky (1995) y Luria (1976) de la necesidad de que la psicología enfrente el problema de la relación entre esas condiciones y formas de conciencia.

Aunque Martín Baró no trabajó directamente el tema de la alfabetización crítica, su trabajo sintetiza, al tiempo que magnifica, los aportes de Vygotski y Freire antes examinados. La tarea de síntesis se resume en la forma en que manejó la relación lenguaje-conciencia conceptualizada por Vygotski y la forma de mediar en dicha relación ideada por Freire en su método de alfabetización y su pedagogía del oprimido. Su apropiación de la concepción vygotskiana del lenguaje como herramienta mediadora en la configuración cognitivo-afectiva que se expresa en la actividad humana está claramente articulada en su texto Psicología social (Martín Baró, 1982). En este, discute la importancia de las palabras en la conformación de la conciencia y hace referencia directa a los aportes de Vygotski $(1991,1993)$ y Luria $(1961,1976)$, a la comprensión de la relación entre lenguaje y pensamiento.

En el mismo texto, Martín Baró amplía la concepción de Vygotski y Luria con respecto a la relación entre lenguaje y conciencia, la cual puede parecer algo abstracta en la formulación original. Esta ampliación la logra al introducir y ejemplificar, en el análisis de esa relación, aspectos sociolingüísticos pertinentes a la educación. Este análisis está orientado por el trabajo de Basil Bernstein (1971) con respecto a la relación entre códigos lingüísticos, clase social y formas de control. Al relacionar estos asuntos y destacar su importancia en impulsar ciertas ideologías, Martín Baró anticipó un movimiento contemporáneo entre estudiosos del enfoque históricocultural que ha puesto en diálogo las ideas Vygotski, Luria y Bernstein (Castlenouvo y Kotik-Friedgut, 2015; Daniels, 2001, 2012). Es interesante notar que una vez examina e integra las propuestas conceptuales de estos tres autores, Martín Baró pasa a vincularlas con la alfabetización crítica de Freire, con lo que amplifica y consolida la red conceptual que sostiene la alfabetización crítica.

Martín Baró (1982) destaca que Bernstein tuvo una "importante intuición sobre el papel mediador del lenguaje entre la estructura social y el desarrollo psicosocial de las personas" (p. 205). Plantea que el problema de la llamada deserción escolar pone de manifiesto "una indudable vinculación lingüística entre escuela y estructura social discriminante" (p. 203). Esta vinculación persiste en análisis contemporáneos de gran pertinencia para la práctica educativa (Baquero, 2016; Baquero, Dicker y Frigerio, 2007). Para Martín Baró se trata de una estructura social en que la institución escolar utiliza códigos lingüísticos para establecer 
límites al curso del desarrollo de sujetos en condición de pobreza y desigualdad. La siguiente cita resume su posición:

El hecho que el sistema de la alfabetización liberadora de Paulo Freire (1971) tenga como pilar la búsqueda de palabras generadoras, propias de la comunidad, parece validar la conexión entre significaciones y estructuras sociales y, por consiguiente, la existencia de códigos lingüísticos que canalizarían socialmente los valores socialmente impuestos. (p. 203)

La conciencia de la relación lengua, ideología y poder es central a dos conceptos claves de la propuesta freiriana: problematización y concienciación. Ambos conceptos son incorporados e integrados a la psicología de la liberación formulada por Martín Baró. Sin embargo, es importante precisar que Vygotski, Freire y Martín Baró no buscaron reducir el mundo a la actividad lingüística, ya que los tres reconocieron la centralidad de las condiciones materiales de vida y la existencia de lo que no puede aprehenderse en la palabra (imaginación, fantasía, configuraciones subjetivas, emociones y afectos). De ahí el reclamo por situar los significados en contexto, interrogarlos, transformarlos en una construcción permanente de sentido a nivel subjetivo e intersubjetivo. Ese reclamo en la obra de Marín Baró se refleja en su análisis de la propaganda, los medios de comunicación pública y sus usos en la formación de opinión pública (Martín Baró, 1983). Se trata de tres temas centrales en la alfabetización crítica actual, sea en formato papel o en formato digital.

En la psicología de la liberación este reclamo se encarna en una práctica profesional crítica y reflexiva asentada en un compromiso ético político. Se plantea como inevitable el tener que hacer una elección con respecto a cómo se produce y utiliza el conocimiento en la disciplina, e igualmente importante cómo se aplica. ¿Cuál es la postura del sujeto que se emplea en el quehacer psicológico ante las condiciones las carencias en la alimentación, la vivienda, la salud y la educación a la hora de apoyar a otros en la comprensión de sus causas y razones? ¿Cómo le apoya en el proceso de entender y manejar las múltiples formas de violencia? ¿Cómo comparte herramientas para ayudar a otros a entender que lo que presentan los medios de comunicación masiva no es información neutral y a reflexionar sobre su efecto en la opinión pública? Esta última pregunta plantea un tema de gran actualidad frente al manejo de nuevas tecnologías, en particular, las redes sociales, como demuestran acontecimientos recientes en el panorama sociopolítico global. Ante los roles 
tradicionalmente asignados para mantener el status quo, Martín Baró pensó en roles posibles para la transformación social (Ratner, 2015).

El trabajo de síntesis y amplificación del legado de Vygotski y de Freire, que se observa en la obra de Martín Baró, puede representarse metafóricamente con un préstamo a la música. Su discurso es in crescendo. En el primer tono el lenguaje es una herramienta para conformar la conciencia. En el segundo tono el lenguaje es una herramienta para interpelar la conciencia. En el tercero es modo de ejecutar la acción consciente mediante la denuncia y la acción militante a cualquier costo. Sin pretender simplificar lo examinado, y en aras de ofrecer destacar aspectos claves del legado de cada uno de los autores examinados, la Tabla 1 presenta una sinopsis de los aportes de Vygotski, Freire y Martín Baró para la alfabetización crítica.

Tabla 1

Sinopsis de aportes de Vygotski, Freire y Martín Baró pertinentes a la alfabetización crítica

\begin{tabular}{|c|c|c|c|c|c|}
\hline & \multicolumn{5}{|c|}{ Categorías de análisis } \\
\hline Autor & Lenguaje & $\begin{array}{c}\text { Actividad } \\
\text { comunicativa }\end{array}$ & Conciencia & $\begin{array}{c}\text { Principales } \\
\text { influencias } \\
\text { filosóficas }\end{array}$ & $\begin{array}{c}\text { Alfabetización/Acción } \\
\text { transformadora }\end{array}$ \\
\hline $\begin{array}{l}\text { Lev S. } \\
\text { Vygotski }\end{array}$ & 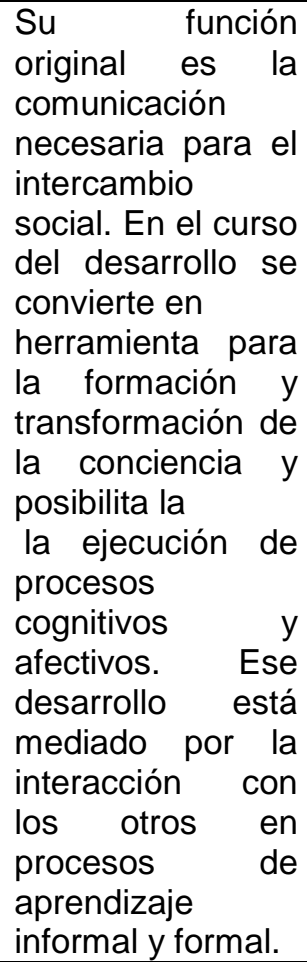 & $\begin{array}{lr}\text { Ocurre } & \text { en } \\
\text { contextos } & \\
\text { específicos } & \text { de } \\
\text { actividad. } & \text { Está } \\
\text { atravesada por las } \\
\text { particulares } \\
\text { situaciones } \\
\text { sociales } \\
\text { desarrollo } \\
\text { aprendizaje en las } \\
\text { que se adquieren } \\
\text { significados y se } \\
\text { construyen } \\
\text { sentidos. }\end{array}$ & $\begin{array}{lr}\text { Emerge en el } \\
\text { curso } \\
\text { prácticas } \\
\text { sociales } \\
\text { históricamente } \\
\text { situadas } \\
\text { culturalmente } \\
\text { mediadas. El } \\
\text { lenguaje es uno } \\
\text { de los } \\
\text { mediadores de } \\
\text { mayor impacto } \\
\text { en su } \\
\text { configuración. }\end{array}$ & $\begin{array}{l}\text { K. Marx } \\
\text { F. Engels } \\
\text { B. Spinoza }\end{array}$ & $\begin{array}{lr}\text { El aprendizaje de la } \\
\text { lectoescritura es } \\
\text { clave en la } \\
\text { transformación de } \\
\text { las funciones } \\
\text { mentales } \\
\text { elementales } \\
\text { complejas y está } \\
\text { atado al desarrollo } \\
\text { de formas de } \\
\text { conciencia. }\end{array}$ \\
\hline
\end{tabular}




\begin{tabular}{|c|c|c|c|c|c|}
\hline Paulo Freire & $\begin{array}{lr}\begin{array}{lr}\text { Como } \\
\text { herramienta }\end{array} \\
\text { para de } \\
\text { construcción de } \\
\text { pensamientos y } \\
\text { afectos, } \\
\text { lenguaje no el } \\
\text { neutral. Lleva } \\
\text { consigo todo un } \\
\text { bagaje de } \\
\text { ideologías que no } \\
\text { se cuestionan en } \\
\text { la educación } \\
\text { bancaria y que la } \\
\text { educación } \\
\text { problematizadora } \\
\text { busca develar. }\end{array}$ & $\begin{array}{l}\text { El diálogo sobre } \\
\text { los significados } \\
\text { contribuye a } \\
\text { develar la relación } \\
\text { entre la palabra y } \\
\text { la realidad. }\end{array}$ & $\begin{array}{l}\text { Su constitución y } \\
\text { expresiones } \\
\text { dependen de la } \\
\text { educación. La } \\
\text { conciencia } \\
\text { crítica depende } \\
\text { de la educación } \\
\text { basada en el } \\
\text { diálogo } \\
\text { problematizador. }\end{array}$ & $\begin{array}{l}\text { K. Marx } \\
\text { Escuela de } \\
\text { Frankfurt/ teoría } \\
\text { crítica }\end{array}$ & $\begin{array}{l}\text { Mediante la } \\
\text { alfabetización crítica, } \\
\text { que toma en cuenta } \\
\text { las situaciones } \\
\text { sociales particulares } \\
\text { de cada sujeto, el ser } \\
\text { humano es capaz de } \\
\text { transformarse a sí } \\
\text { mismo y, con ello, } \\
\text { sus condiciones de } \\
\text { vida. Esta acción } \\
\text { transformadora es } \\
\text { praxis que se ancla } \\
\text { en los procesos de } \\
\text { problematización y } \\
\text { concienciación en la } \\
\text { educación } \\
\text { liberadora. }\end{array}$ \\
\hline $\begin{array}{c}\text { Ignacio } \\
\text { Martín Baró }\end{array}$ & $\begin{array}{l}\text { El lenguaje porta } \\
\text { ideologías y es un } \\
\text { mediador entre la } \\
\text { estructura social y } \\
\text { el desarrollo } \\
\text { psicosocial de las } \\
\text { personas que } \\
\text { debe ser } \\
\text { interpelado. }\end{array}$ & $\begin{array}{l}\text { Los actos de } \\
\text { comunicación en } \\
\text { la educación } \\
\text { deben orientarse a } \\
\text { desideologizar la } \\
\text { experiencia de la } \\
\text { vida cotidiana y } \\
\text { hacer evidente la } \\
\text { relación entre } \\
\text { lengua, ideología y } \\
\text { poder } \\
\text { ejemplificada en el } \\
\text { uso de medios de } \\
\text { comunicación } \\
\text { masiva. }\end{array}$ & $\begin{array}{l}\text { La acción } \\
\text { consciente se } \\
\text { asienta en la } \\
\text { problematización } \\
\text { que lleva a la } \\
\text { denuncia y la } \\
\text { acción militante. }\end{array}$ & $\begin{array}{l}\text { K. Marx } \\
\text { Escuela de } \\
\text { Frankfurt } \\
\text { Teología de la } \\
\text { liberación } \\
\text { Paulo Freire }\end{array}$ & $\begin{array}{l}\text { La alfabetización } \\
\text { concienciadora es } \\
\text { una respuesta } \\
\text { histórica a la } \\
\text { carencia de palabra } \\
\text { personal y social al } \\
\text { ofrecer herramientas } \\
\text { para "leer" nuestra } \\
\text { realidad. Es clave en } \\
\text { el desarrollo de } \\
\text { prácticas sociales y } \\
\text { profesionales } \\
\text { reflexivas orientadas } \\
\text { por el compromiso } \\
\text { ético-político, la } \\
\text { justicia y la equidad. }\end{array}$ \\
\hline
\end{tabular}

Fuente: Elaboración propia, 2018

\section{Vigencia y movimiento en los aportes}

La vigencia de la obra de Vygotski, Freire y Martín Baró se observa en varios frentes. Primero, en la continua reedición de sus obras y en antologías de obras selectas en formato de libro tradicional. Esto se ha realizado tanto en el idioma de publicación original como en traducciones a múltiples idiomas, lo cual ha contribuido de manera significativa a la difusión de sus ideas y la apropiación y adaptación de estas a otras realidades. Segundo, la vigencia se observa en la cantidad de portales electrónicos y enlaces orientados a la divulgación de sus textos en el medio digital. Esto puede constatarse fácilmente al escribir los nombres de los referidos autores en los mecanismos de búsqueda de internet. Finalmente, también se observa la vigencia en la notable cantidad de publicaciones académicas que se generan a partir de su obra. Esto incluye biografías, interpretaciones y elaboraciones. Entre estas 
últimas destacan nuevos conceptos fundamentados en su aportes y aplicaciones a nuevas realidades, como la alfabetización digital. Lo anterior evidencia que la obra de los tres autores examinados son letra viva.

En cuanto al movimiento, en los aportes de Vygotski, Freire y Marín Baró, la propia noción de movilidad evoca la idea de palabra generadora de Freire en dos sentidos. En primer lugar, en la creación de conceptos que magnifican la red conceptual originaria del legado y aplicaciones relacionadas. En segundo lugar, en producciones que enfocan directamente puntos de encuentro y desencuentro en sus ideas o reclaman reflexividad histórica con respecto a lecturas e interpretaciones de sus obras. Es imposible, en un trabajo de esta naturaleza, dar cuenta detallada de las dos instancias mencionadas de movimiento, pero procede mencionar algunos como ejemplo.

Portes (2005) y Portes y Salas (2011) se han ocupado de examinar las aplicaciones de las ideas aquí discutidas, con énfasis en las ideas de Vygotski, a la situación de inequidad en la educación en los Estados Unidos de Norteamérica. Sus aproximaciones transitan desde la denuncia de la inequidad hacia a las alternativas de acción social (con particular peso en la acción educativa) y transformación orientada a la justicia social. La educación es clave en estas elaboraciones, como también lo es en el desarrollo de nuevos conceptos que se asientan tanto en las ideas de Vygotski como en las de Freire. Es el caso de las nociones de fondos de conocimiento y fondos de identidad como nuevos conceptos (Esteban-Guitart, 2016; Esteban-Guitart, Moll, 2014a, 2014b). También es el caso de la aplicación de las ideas a instancia de aprendizaje, como es el caso de la enseñanza de un idioma dominante en el contexto de búsqueda de justicia social (Souto-Manning y Smagorinsky, 2011).

Más allá de la institución educativa y el trabajo en las aulas, se observa vigencia y movimiento en aplicaciones a la psicología clínica, la psicoterapia y a procesos de supervisión en la formación en dichas áreas. De particular interés, porque integran conceptos claves de los tres autores referidos, son las que conciernen a intervenciones con poblaciones minoritarias y de idioma diferente en la sociedad que les alberga, como es el caso de los inmigrantes en los Estados Unidos de Norteamérica (Torres, Maldonado y Alarcón, 2013). Hay esfuerzos importantes por orientar las mencionadas áreas de trabajo por los principios de la psicología de la liberación y documentar la efectividad de su aplicación. Según Tate, Torres Rivera, Brown y Staistis (2013), dicha orientación se caracteriza por aceptar la crítica de Martín Baró a la psicología, e impulsar su proyecto de cambio, reconocer y recuperar la memoria histórica de las personas y grupos con los que se trabaja, desideologizar su 
experiencia cotidiana, sostener y utilizar sus virtudes y fortalezas, y apoyar sus procesos de problematización y concienciación.

El ejemplo final de movimiento es el más reciente, y algunos autores reclaman que las vertientes críticas en la psicología y la educación han sido lentas en enfrentar los problemas que las nuevas tecnologías de la información y la comunicación han planteado (Landow, 2006). El uso de estas tecnologías ha introducido variantes en el lenguaje con la creación de códigos lingüísticos compartidos por los usuarios e imbricados con imágenes como lo "emojis". También se han documentado cambios en procesos cognitivos en usuarios frecuentes (Rodríguez Arocho, 2018). Igualmente, se ha documentado la movilización y manipulación de fuerzas sociopolíticas en las redes sociales. Lo anterior, impone retos a la educación y la psicología que los aportes de Vygotski, Freire y Martín Baró pueden ayudar a enfrentar, particularmente en lo que concierne a la alfabetización crítica (McLaren y Jandric, 2013).

Parece una tarea importante el aproximarse a las nuevas significaciones para entenderlas antes de problematizarlas. La problematización implicaría formular preguntas como ¿cuáles son los nuevos medios y prácticas de alfabetización?, ¿cuál es su historia y cómo afectan nuestras prácticas sociales?, ¿quién, cómo y con qué propósitos determina qué información circula en la internet y hacia quién? y ¿cómo promover la conciencia crítica en las condiciones de opresión o liberación que posibilitan los nuevos artefactos culturales? Estas preguntas generadoras se orientan a la concienciación y la desideologización en los términos planteados por los autores examinados.

La actual literatura documenta que han comenzado esfuerzos por entender las variantes en alfabetización y sus relaciones con el mundo en que se vive (Mills, 2016). Estos esfuerzos podrían fortalecerse con el intercambio de ideas y acciones conjuntas de la psicología de la liberación y la pedagogía crítica en el contexto de las actuales dinámicas económicas y políticas (Dobles Oropeza, Baltodano Arróliga y Leandro Zúñiga, 2007; Rodríguez Arocho, 2010). Ese intercambio es una tarea incompleta en la psicología y la educación que puede transformarse en agenda de futuro.

\section{Síntesis y conclusión}

La producción, distribución y uso de conocimientos ha estado atravesada por historia de la alfabetización, una práctica social mediante la cual aprendemos a codificar y decodificar el lenguaje escrito. Hemos argumentado que esa práctica puede realizarse Volumen 19 Número 1 (Enero-Abril), ISSN 1409-4703 
irreflexivamente o con conciencia crítica. Las ideas y las prácticas desarrolladas por Vygotski, Freire y Martín Baró pueden articularse como base conceptual para la práctica de una alfabetización crítica. Vygotski, con la colaboración de Luria, se ocupó de demostrar que la apropiación y uso del lenguaje escrito modifica los procesos psíquicos asociados con la actividad consciente propiamente humana. Se ocupó también de apuntar al significado de la palabra como la unidad entre lenguaje y pensamiento, con la advertencia de que ese pensamiento solo puede comprenderse cabalmente si se entienden las circunstancias, los motivos, los intereses y los sentimientos del sujeto que enuncia la palabra. Por su parte, Freire se ocupó de desarrollar un método para hurgar en esos significados, problematizarlos y promover el desarrollo de una conciencia crítica que se exprese en la reflexión y en actividad transformadora. Martín Baró, a partir de estas dos ideas, subrayó el papel de la ideología en la configuración de significados, y concibió una psicología de la liberación fundamentada en la desideologización, la concienciación y el compromiso ético-político en la búsqueda de la equidad y la justicia social.

A partir del examen realizado, podemos concluir dos cosas con relación a la vigencia y al movimiento de las obras de Vygotski, Freire y Martín Baró. En primer lugar, la obra de estos autores es vigente dado que algunos de los problemas sobre los que teorizaron e investigaron no han desaparecido. En algunos casos, se expresan de la misma forma que cuando fueron planteados, y en otros aparecen transformados, como se observa en los indicadores analfabetismo tradicional y analfabetismo digital. Este último ha resultado en elaboraciones complejas en la que el texto no necesariamente está escrito en papel sino expresado en imágenes. Nuevos conceptos como hipertexto e intertextualidad reflejan cambios significativos en las formas de lectoescritura. A pesar de estos cambios en contenido y en forma, los principios de la alfabetización crítica se mantienen firmes invitando a su problematización y a la reflexión crítica con respecto a la alfabetización digital y las configuraciones de mente que posibilitan.

En segundo lugar, el movimiento de su legado se observa en la creación de nuevos conceptos y en su aplicación, tanto al campo de la psicología como al de la educación. La teorización y la investigación sobre el legado de Vygotski, Freire y Martín Baró sigue vigente. Entre estos hay trabajos que rescatan la idea de tomar la comunidad como punto de partida, de reconocer y utilizar el saber que en ella se produce, considerar las condiciones materiales de vida (particularmente las relativas a la pobreza y la marginalización y tomar parte activa en los esfuerzos de transformación social mediante una práctica académica y profesional con 
compromiso ético-político. Esto cobra un significado especial ante las actuales dinámicas históricoculturales, socioeconómicas y geopolíticas, y sirve de fundamento al compromiso ético político por el que abogó Ignacio Martín Baró.

\section{Referencias}

Aaron, Adrianne and Corne, Shawn (1994). Writings for a liberation of psychology: Ignacio Martín Baró. Cambridge, MA: Harvard University Press.

Baquero, Ricardo (2016). De las dificultades de predecir: el fracaso escolar en los enfoques socioculturales. En Wanda C. Rodríguez Arocho, Maurizia D'Antoni and Victoria González (Eds.), Vygotski y su legado a la investigación en América Latina (pp. 9-24). San José: Instituto de Investigación en Educación de la Universidad de Costa Rica.

Baquero, Ricardo, Dicker, Gabriela and Frigerio, Graciela (Eds.). (2007). Las formas de lo escolar. Buenos Aires: del Estante Editorial.

Blanck, Guillermo. (1990). Vygotsky: The man and his cause. In Luis Moll (Ed.), Vygotsky and education: Instructional implications and applications of sociohistorical psychology (pp. 31-58). Cambridge: Cambridge University Press.

Bernstein, Basil. (1971). Class, codes and control (Vol. I). London: Routledge.

Blanco, Amalio, de la Corte, Luis and Sabucedo, José Manuel (2018). Para una psicología social crítica no construccionista: reflexiones a partir del realismo crítico de Ignacio Martín Baró. Univerisitas Pyschologica, 17(1), 1-25.

Bruner, Jerome S. (1962). Prologue. Thought and Language. Cambridge, MA: MIT Press.

Bruner, Jerome S. (1987). Prologue. In Robert W. Rieber y Aaron Carton (Eds.). The collected works of L. S. Vygotsky (pp. 1-16). New York Plenun Press.

Burke, James and Ornstein, Robert. (1995). Del hacha al chip: Cómo la tecnología cambia nuestras mentes. Barcelona: Editorial Planeta.

Castlenouvo. Antonella and Kotik-Friedgut, Bella. (2015). Vygotsky and Bernstein in the light of Jewish tradition. Brigton, MA: Academic Studies Press.

Clark, Andy. (2003). Natural-born cyborgs: Minds, technologies and the future of human intelligence. New York: Oxford University Press.

Cole, Michael. (1996). Cultural psychology. Cambridge, MA: Harvard University Press.

Colombo, Fausto and Fortunati, Leopoldina (Eds.). (2011). Broadband society and generational changes. New York: Peter Lang.

Daniels, Harry. (2001). Vygotsky and pedagogy. New York: Routledge. 
Daniels, Harry (Ed.) (2012). Vygotsky and sociology. New York: Routledge.

Dobles Oropeza, Ignacio, Baltodano Arróliga, Sara and Leandro Zúñiga, Vilma (Eds.). (2007). Psicología de la liberación en el contexto de la globalización neoliberal: Acciones, reflexiones y desafíos. San José: Editorial de la Universidad de Costa Rica.

Esteban-Guitart, Moisés. (2013). Principios y aplicaciones de la psicología cultural: Una aproximación vygotskiana. Madrid: Miño y Dávila.

Esteban-Guitart, Moisés. (2016). Funds of identity: Connecting meaningful learning experiences in and out schools. Cambridge: Cambridge University Press.

Esteban-Guitart, Moisés and Moll, Luis. (2014a). Funds of identity: a new concept based on funds of knowledege approach. Culture and Psychology, 20(1), 31-48.

Esteban-Guitart, Moisés and Moll, Luis. (2014b). Lived expriences, funds of identity and education. Culture and Psychology. Culture and Psychology, 20(1), 70-81.

Flores, Leonardo. (2018). La alfabetización: evoluciones, revoluciones y vestigios. 80 Grados. Recuperado de http://www.80grados.net/la-alfabetizacion-evolucionesrevoluciones-y-vestigios/

Freire, Paulo. (1971). La educación como práctica de la libertad. Motenvideo: Tierra Nueva.

Freire, Paulo. (1972). Pedagogía del oprimido. Buenos Aires: Editorial Siglo XXI.

Freire, Paulo. (1991). La importancia del acto de leer y el proceso de libración. México: Editorial Siglo XXI.

Freire, Paulo and Macedo, Donaldo. (1987). Literacy: Reading the word and the world. New York: Taylor and Francis.

Figueroa Sarriera, Heidi. (2017). Imaginarios del sujeto en la era digital: Post (identidades) contemporáneas. Quito, Ecuador: Ediciones CIESPAL.

Gerdhart, Heinz-Peter. (1993). Paulo Freire (1921-1997). Perspectivas: revista trimestral de educación comparada, 23(3/4), 439-458. Paris: UNESCO. Recuperado de http://www.ibe.unesco.org/sites/default/files/freiree.PDF

González Rey, Fernando Luis. (2011). El pensamiento de Vygotsky: Contradicciones, desdoblamientos y desarrollos. México: Editorial Trillas.

Kiincheloe, Joe L. (2008). Critical pedagogy (2 ${ }^{\text {nd }}$. ed.). New York: Peter Lang.

Kirylo, James. (2011). Paulo Freire: The man from Recife. New York: Peter Lang.

Kozulin, Alex. (2000). Instrumentos psicológicos: La educación desde la perspectiva sociocultural. Buenos Aires: Paidós. 
Kozulin, Alex, Gindis, Boris, Ageyev, Vladimir S. and Miller, Suzanne (Eds.). (2003). Vygotsky's educational theory in cultural context. New York: Cambridge University Press.

Lamdan, Eli and Yasnitsky, Anton. (2016). Do Uzbek's have illusions? The Luria-Koffka controversy of 1932. In Anton Yasnitsky and René Van der Veer (Eds), Revisionist revolution in Vygotsky studies (pp. 172-200). New York: Routledge.

Landow, George. (2006). Hypertext 3.0: critical theory and new media in an era of globalization. Baltimore: The John Hopkins University Press.

Lake, Robert. (2012). Vygotsky on education. New York: Peter Lang Publishing.

Lee, Carol and Smagorinsky, Peter (Eds.). (2001). Vygotskian perspectives on literacy research: Constructing meaning trough collaborative inquiry. New York: Cambridge University Press.

Luria, Alexander Romanovich. (1961). The role of speech in the regulation of normal and abnormal behavior. London, Pargamon.

Luria, Alexander Romanovich. (1976). Cognitive development: is cultural and social foundations. Cambridge, MA: Harvard University Press.

Martín Baró, Ignacio. (1982). Psicología social II. San Salvador. UCA Editores.

Martín Baró, Ignacio. (1983). Acción e ideología: psicología social desde Centroamérica. San Salvador: UCA Editores.

Martín Baró, Ignacio. (1986). Hacia una psicología de la liberación. Boletín de PsicologíaUniversidad Centroamericana, (22), 219-231.

McLaren, Peter and Jandric, Petar. (2014). Critical revolutionary pedagogy is made by walking: in a world where many worlds coexist. Policy Futures in Education, 12(6). http://journals.sagepub.com/doi/pdf/10.2304/pfie.2014.12.6.805

Mills, Kathy. (2016). Literacy theories for the digital age: Social, critical, multimodal, spatial, material and sensory lenses. New York: Multilingual Matters.

Moll, Luis (Ed.). (1990). Vygotsky and education: Instructional implications and applications of sociohistorical psychology (pp. 31-58). Cambridge: Cambridge University Press.

Moll, Luis. (2014). L.S. Vygotsky and education. New York: Taylor and Francis.

Montero, Maritza. (1991). Psicología de la liberación: Propuesta para una teoría sociopsicológica. En H. Riquelme (Ed.), Otras realidades, otras vías de acceso (pp. 133-150). Caracas: Nueva Sociedad.

Montero, Maritza. (2004). Relaciones entre psicología social comunitaria, psicología crítica y psicología de la liberación: Una respuesta latinoamericana. Psyche, 13(2), 17-28. 
Olson, David. (1994). The world on paper: the conceptual and cognitive implications of reading and writing. New York: Cambridge University Press.

Olson, David and Cole, Michael (Eds.). (2006). Technology, literacy, and the evolution of society: Implications of the work of Jack Goody. New York: Psychology Press.

Ojokheta, K. O. (2007). Paulo Freire's literacy teaching methodology. International Journal of Adult Education and Development. Recuperado de https://www.dvvinternational.de/adult-education-and-development/editions/aed-692007/10thanniversary-of-paulo-freirersquos-death/paulo-freirersquos-literacy-teachingmethodology/

Portes, Pedro. (2005). Dismantling educational inequality: a cultural historical approach to closing the achievement gap. New York: Peter Lang Publishing.

Portes, Pedro and Salas, Spencer (Eds.). (2011). Vygotsky in the 21st century: advances in theory and practice with non-dominant communities. New York: Peter Lang.

Portillo, Nelson. (2012). The life of Ignacio Martín Baró: a narrative account of a personal biographical journey. Peace and Conflict, 18(1), 77-87.

Ratner, Carl. (2015). Recuperación y promoción sobre las ideas de Ignacio Martín Baró sobre psicología, cultura y transformación social. Teoría y Crítica de la Psicología, (6). Recuperado de http://www.teocripsi.com/ojs/index.php/TCP/article/view/29

Rodríguez Arocho, Wanda C. (2000). El tema de la conciencia en la psicología de Vygotski y en la pedagogía de Freire: Implicaciones para la educación. In Ángel Villarini, F. Noriega and D. Shipiani (Eds.), Globalización, pensamiento crítico y pedagogía de la liberación: Actas del 10mo. Encuentro Nacional de Educación y Pensamiento (pp. 178192). San Juan: Universidad de Puerto Rico. Recuperado de https://es.scribd.com/document/26995610/EL-TEMA-DE-LA-CONCIENCIA-EN-LAPSICOLOGIA-DEVigostky-y-freire

Rodríguez Arocho, Wanda C. (2010). Psicología de la liberación y pedagogía crítica: Un examen de sus aportes y retos. Pedagogía, 43(1), 13-34.

Rodríguez Arocho, Wanda C. (2013). El lugar de la afectividad en la psicología de Vygotski: Reflexividad histórica y reivindicación. Propósitos y Representaciones, 1(2), 105-129

Rodríguez Arocho, Wanda C. (2015). Reflexividad histórica, problematización e indagación dialógicas como herramientas para repensar el concepto vygotskiano de zona de desarrollo próximo. Revista Puertorriqueña de Psicología, 26(1), 10-24. Recuperado de http://www.redalyc.org/pdf/2332/233245620002.pdf

Rodríguez Arocho, Wanda C. (2018). Herramientas culturales y transformaciones mentales: Las tecnologías de la información y la comunicación en perspectiva histórico-cultural. Actualidades Investigativas en Educación, 18(2), 1-21. https://revistas.ucr.ac.cr/index.php/aie/article/view/33068/32543

Rojas Osorio, Carlos. (1998). Dimensiones del pensamiento crítico. Crecemos, 4(1), 9-14. Volumen 19 Número 1 (Enero-Abril), ISSN 1409-4703 
Sabila, Paula. (2009). El hombre postorgánico: Cuerpo, subjetividad y tecnologías digitales. Buenos Aires: Fondo de Cultura Económica.

Smagorkinsky, Peter. (2011). Vygotsky and literacy research: A methodological framework. The Netherlands: Sense Publishers.

Souto-Manning, Mariana and Smagorinsky, Peter. (2011). Freire, Vygotsky and social justice theories in English education. In S.J. Miller and David Kirkland (Eds). Change matters: Critical essays on moving social justice research to theory to policy. New York: Peter Lang.

Tate, Kevin, Torres Rivera, Edil, Brown, Eric and Skaistis, Leslie. (2013). Foundations for Liberation: Social Justice, Liberation Psychology, and Counseling. Revista Interamericana de Psicología, 47(3), 373-382.

Torres, Edil, Maldonado, José and Alarcón, Laura. (2013). From Vygotsky to Martín Baró: dealing with language and liberation during the supervision process. Universal Journal of Psychology, 1(2), 32-40.

Van der Veer, René and Valsiner, Jaan. (1991). Understanding Vygotsky: A quest for synthesis. Cambridge, MA: Blackwell.

Villacañas de Castro, Luis Sebastián. (2016). Critical pedagogy and Marx, Vygotsky and Freire: Phenomenal forms and educational action research. New York: Palgrave McMillan.

Vygotski, Lev S. (1991). El significado histórico de la crisis en la psicología. En L. S. Vygotski: Obras Escogidas (Tomo I; pp. 257-407). Madrid. Aprendizaje Visor.

Vygotski, Lev S. (1993). Pensamiento y lenguaje. En Lev S. Vygotsky: Obras Escogidas (Tomo II; pp. 9-348). Madrid. Aprendizaje Visor.

Vygotski, Lev S. (1995). Historia del desarrollo de las funciones psíquicas superiores (Obras Escogidas III). Madrid: Aprendizaje Visor.

Vygotsky, Lev S. and Luria, Alexander. (1994). Tool and symbol y child development. In René Van de Veer and J. Jaan Valsiner (Eds,), The Vygotsky reader (pp. 99-174). Cambridge, MA: Blackwell.

Yaroshevsky, Mikhail. (1989). Lev Vygotsky. Moscow: Progress Press.

Yasnitsky, Anton, Van der Veer, René and Ferrari, Michael (2014). (Eds.). Cambridge handbook of cultural historical psychology. New York: Cambridge University Press. 